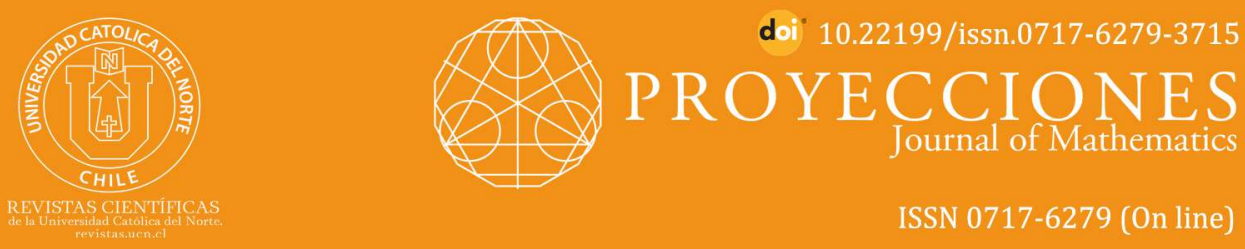

\title{
Total irregularity strength of some cubic graphs
}

Muhammad Ibrahim ${ }^{1}$ orcid.org/0000-0002-2272-6342

S. Khan ${ }^{2}$

Muhammad Ahsan Asim³ ${ }^{3}$ orcid.org/0002-4215-2825

Muhammad Waseem ${ }^{4}$ () orcid.org/0000-0001-9442-5664

Bahauddin Zakariya University, Centre for Advanced Studies in Pure and Applied Mathematics, Multan, Pakistan.

$1 \square$ mibtufail@gmail.com; ${ }^{2 \square}$ sanamath07@gmail.com; ${ }^{\square}$ muhammadwaseemakram00@gmail.com ${ }^{3}$ Jazan University, Fcs. of Computer Science \& Info. Technology, Jazan, Kingdom of Saudi Arabia. mr.ahsan.asim@gmail.com

Received: 2019/08/19 | Accepted: 2021/01/21

Abstract:

Let $G=(V, E)$ be a graph. A total labeling $\psi: V \cup E \rightarrow\{1,2, \ldots, k\}$ is called totally irregular total $k$-labeling of $G$ if every two distinct vertices $u$ and $v$ in $V(G)$ satisfy $w t(u) \neq w t(v)$, and every two distinct edges $u_{1} u_{2}$ and $v_{1} v_{2}$ in $E(G)$ satisfy $w t\left(u_{1} u_{2}\right) \neq w t\left(v_{1} v_{2}\right)$, where $w t(u)=\psi(u)+\sum_{u v \in E(G)} \psi(u v)$ and $w t\left(u_{1} u_{2}\right)=\psi\left(u_{1}\right)+\psi\left(u_{1} u_{2}\right)+$ $\psi\left(u_{2}\right)$. The minimum $k$ for which a graph $G$ has a totally irregular total $k$-labeling is called the total irregularity strength of $G$, denoted by $\operatorname{ts}(G)$.

In this paper, we determine the exact value of the total irregularity strength of cubic graphs.

Keywords: Total edge irregularity strength; Total vertex irregularity strength; Total irregularity strength; Plane graph; Crossed prism graph; Necklace graph; Goldberg Snark graph.

MSC (2020): 05C78, 90C35, 90C27.

\section{Cite this article as (IEEE citation style):}

M. Ibrahim, M. A. Asim, S. Khan, and M. Waseem, "Total irregularity strength of some cubic graphs", Proyecciones (Antofagasta, On line), vol. 40, no. 4, pp. 905-918, 2021, doi: 10.22199/issn.0717-6279-3715

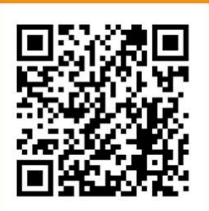

Article copyright: (C) 2021 Muhammad Ibrahim, Muhammad Ahsan Asim, S. Khan and Muhammad Waseem. This is an open access article distributed under the terms of the Creative Commons License, which permits unrestricted use and distribution provided the original author and source are credited. 


\section{Introduction}

As a standard notation, assume that $G=G(V, E)$ is a finite, simple and undirected graph with $p$ vertices and $q$ edges. A labeling of a graph is any mapping that sends some set of graph elements to a set of numbers (usually positive integers). if the domain is the vertex-set or the edge-set, the labeling are called respectively vertex-labeling or edge labeling. If the domain is $V \cup E$ then we call the labeling a total labeling. In many cases it is interesting to consider the sum of all labels associated with a graph element. This will be called the weight of element.

For a graph $G$ we define a labeling $\phi: V \cup E \rightarrow\{1,2, \ldots, k\}$ to be a total $k$ - labeling. A total $k$-labeling $\phi$ is defined to be an edge irregular total $k$-labeling of the graph $G$ if for every two different edges $u v$ and $u^{\prime} v^{\prime}$ their weights $\phi(u)+\phi(u v)+\phi(v)$ and $\phi\left(u^{\prime}\right)+\phi\left(u^{\prime} v^{\prime}\right)+\phi\left(v^{\prime}\right)$ are distinct. Similarly a total $k$-labeling $\phi$ is defined to be an vertex irregular total $k$-labeling of the graph $G$ if for every two different vertices $u$ and $v$ their weights $w t(u)$ and $w t(v)$ are distinct. Here, the weight of a vertex $x$ in $G$ is the sum of the label of $u$ and the labels of all edges incident with the vertex $u$. The minimum $k$ for which the graph $G$ has an edge irregular total $k$-labeling is called the total irregularity strength of $G$, denoted by tes $(G)$. Analogously, the minimum $k$ for which the graph $G$ has a vertex irregular total $k$-labeling is called the total vertex irregularity strength of $G$, denoted by $\operatorname{tvs}(G)$.

The total edge irregularity strength and total vertex irregularity strength are invariant analogous to irregular assignments and the irregularity strength of a graph $G$ introduced by Chartrand et al. [11] and studied by numerous authors, see $[9,13,14,16,23]$. The irregular assignment is a $k$-labeing of the edge $\phi: E \rightarrow\{1,2, \ldots, k\}$ such that the sum of the labels of edges incident with a vertex is different for all the vertices of $G$, and the smallest $k$ for which there is an irregular assignment is the irregularity strength, denoted by $s(G)$.

A simple lower bound for $\operatorname{tes}(G)$ and $\operatorname{tvs}(G)$ of a $(p, q)$-graph $G$ in terms of maximum degree $\Delta(G)$ and the minimum degree $\delta(G)$, determine in the following theorems.

Theorem 1. [9] Let $G$ be a $(p, q)$ - graph with maximum degree $\Delta=\Delta(G)$ then $\operatorname{tes}(G) \geq \max \left\{\left\lceil\frac{q+2}{3}\right\rceil,\left\lceil\frac{\Delta+1}{2}\right\rceil\right\}$ 
Theorem 2. [9] Let $G$ be a $(p, q)$-graph with minimum degree $\delta=\delta(G)$ and maximum degree $\Delta=\Delta(G)$ then

$$
\left\lceil\frac{p+\delta}{\Delta+1}\right\rceil \leq t v s(G) \leq p+\Delta-2 \delta+1
$$

Ivančo and Jendrol [15] posed the following conjecture:

Conjecture 1. Let $G$ be an arbitrary graph different from $K_{5}$. Then $\operatorname{tes}(G)=\max \left\{\left\lceil\frac{q+2}{3}\right\rceil,\left\lceil\frac{\Delta+1}{2}\right\rceil\right\}$

In [20] Nurdin et al. posed the following conjecture:

Conjecture 2. Let $G$ be a connected graph having $n_{i}$ vertices of degree $i(i=\delta, \delta+1, \delta+2, \ldots, \Delta)$, where $\delta$ and $\Delta$ are the minimum and the maximum degree of $G$ respectively. Then

$$
\operatorname{tvs}(G)=\max \left\{\left\lceil\frac{\delta+n_{\delta}}{\left.\delta+1\rceil,\left[\frac{\delta+n_{\delta}+n_{\delta+1}}{\delta+2}\right\rceil, \ldots,\left[\frac{\delta+\sum_{i=\delta}^{\Delta} n_{i}}{\Delta+1}\right\rceil\right\} .}\right.\right.
$$

Conjecture 1 has been for complete graphs and complete bipartite graphs $[16,17]$, for the grid [19], for hexagonal grid graphs [5], for toroidal grid [12], for generalized prism [10], for categorical product of two cycles [1], for strong product of cycles and paths [6], for zigzag graphs [7] and for strong product of two paths [3].

Conjecture 2 has been verified for trees [20], for circulant graphs [8].

Combining both total edge irregularity strength and total vertex irregularity strength notions, Marzuki et al. [18] introduced a new irregular total $k$-labeling of a graph $G$, which is required to be at the same time both vertex and edge irregular. The minimum value of $k$ for which such labeling exist is called total irregularity strength of graph and is denoted by $t s(G)$. Besides that, they determined the total irregularity strength of cycles and paths. Marzuki, et al. [18] given a lower bond of $t s(G)$ as follows.

$$
\text { For every graph G, } t s(G) \geq \max \{\operatorname{tes}(G), \operatorname{tvs}(G)\}
$$

Ramdani and Salman [21] showed that the lower bound in (1.1) for some cartesian product graphs is tight. In [2], Ahmad et al. found the exact value of total irregularity strength of generalized Petersen graph. 


\section{The plane graph $D_{n}$}

In [4] A. Ahmad et al. defined the plane graph $D_{n}$ and found the vertex irregular total labeling of cubic graphs. we have investigated the total irregularity strength of plane graph, cross prism graph, Necklace graph and goldberg snark graph.

Let $D_{n}$ be a plane graph. The set of vertices and edges of the plane graph $D_{n}$ is given as followed.

$$
\begin{gathered}
V\left(D_{n}\right)=V\left\{a_{i} ; b_{i} ; c_{i} ; d_{i}: 1 \leq i \leq n\right\} \\
E\left(D_{n}\right)=\left\{c_{i} c_{i+1} ; b_{i} c_{i} ; a_{i} b_{i} ; b_{i} d_{i} ; a_{i} d_{i} ; a_{i+1} d_{i}: 1 \leq i \leq n\right\}
\end{gathered}
$$

where the subscript $n+1$ must be replaced by 1 . In the next theorem we determined the total irregularity strength of plane graphs $D_{n}$.

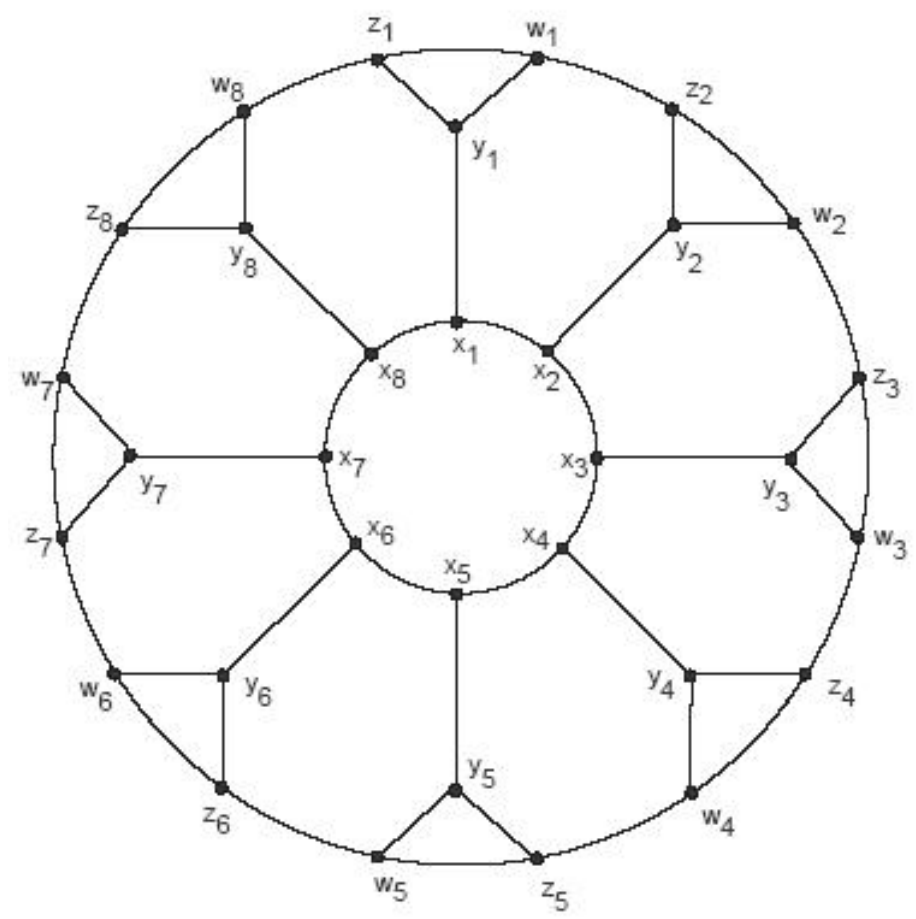

Figure 1: The plane graph $D_{8}$

Theorem 3. Let $D_{n}, n \geq 3$ be plane graph, Then $t s\left(D_{n}\right)=2 n+1$ 
Proof: Since $\left|E\left(D_{n}\right)\right|=6 n$, so from Theorem 1 , tes $\left(D_{n}\right) \geq 2 n+1$. Also $D_{n}$ has $4 n$ vertices of degree 3 , so from Theorem 2 , we get $\operatorname{tvs}\left(D_{n}\right) \geq$ $\left\lceil\frac{4 n+3}{4}\right\rceil$. From equation (1.1), we get $t s\left(D_{n}\right) \geq 2 n+1$ Now we show that $t s\left(D_{n}\right) \leq 2 n+1$. For this we define a total labeling $\phi$ from $V\left(D_{n}\right) \cup E\left(D_{n}\right)$ into $\{1,2, \ldots, 2 n+1\}$ and compute the vertex weight and edge weight in the following way.

For $1 \leq i \leq n$,

$\phi\left(c_{i}\right)=i, \phi\left(b_{i}\right)=1, \phi\left(a_{i}\right)=n+i, \phi\left(d_{i}\right)=k, \phi\left(b_{i} c_{i}\right)=i, \phi\left(b_{i} d_{i}\right)=n+i$, $\phi\left(a_{i} d_{i}\right)=n+1, \phi\left(a_{i+1} d_{i}\right)=k, w t\left(a_{i} d_{i}\right)=4 n+2+i, w t\left(b_{i} d_{i}\right)=3 n+2+i$, $w t\left(b_{i} c_{i}\right)=1+2 i, w t\left(d_{i}\right)=6 n+3+i$,

$w t\left(a_{i+1} d_{i}\right)= \begin{cases}5 n+3+i, & \text { for } 1 \leq i \leq n-1 \\ 5 n+3, & \text { for } i=n\end{cases}$

Case.1. when $n$ is even

$$
\begin{aligned}
& \phi\left(c_{i} c_{i+1}\right)= \begin{cases}1, & \text { for } 1 \leq i \leq n-1 \\
n+2, & \text { for } i=n\end{cases} \\
& \phi\left(a_{i} b_{i}\right)= \begin{cases}n, & \text { for } i=1 \\
n+1, & \text { for } 2 \leq i \leq n\end{cases} \\
& w t\left(c_{i} c_{i+1}\right)= \begin{cases}2+2 i, & \text { for } 1 \leq i \leq n-1 \\
2 n+3, & \text { for } i=n\end{cases} \\
& w t\left(a_{i} b_{i}\right)= \begin{cases}2 n+2, & \text { for } i=1 \\
2 n+2+i, & \text { for } 2 \leq i \leq n\end{cases} \\
& w t\left(a_{i}\right)= \begin{cases}5 n+3, & \text { for } i=1 \\
5 n+3+i, & \text { for } 2 \leq i \leq n\end{cases} \\
& w t\left(b_{i}\right)= \begin{cases}2 n+3, & \text { for } i=1 \\
2 n+2+2 i, & \text { for } 2 \leq i \leq n\end{cases} \\
& w t\left(c_{i}\right)= \begin{cases}n+5, & \text { for } i=1 \\
2+2 i, & \text { for } 2 \leq i \leq n-1 \\
3 n+3, & \text { for } i=n\end{cases}
\end{aligned}
$$

Case.2. when $n$ is odd $\phi\left(a_{i} b_{i}\right)=n+1, w t\left(a_{i} b_{i}\right)=2 n+2+i$, 


$$
\begin{aligned}
& \phi\left(c_{i} c_{i+1}\right)= \begin{cases}1, & \text { for } 1 \leq i \leq n-1 \\
n+1, & \text { for } i=n\end{cases} \\
& w t\left(c_{i} c_{i+1}\right)= \begin{cases}2+2 i, & \text { for } 1 \leq i \leq n-1 \\
2 n+2, & \text { for } i=n\end{cases} \\
& w t\left(b_{i}\right)= \begin{cases}2 n+4, & \text { for } i=1 \\
2 n+2+2 i, & \text { for } 2 \leq i \leq n\end{cases} \\
& w t\left(a_{i}\right)= \begin{cases}5 n+4, & \text { for } i=1 \\
5 n+3+i, & \text { for } 2 \leq i \leq n\end{cases} \\
& w t\left(c_{i}\right)= \begin{cases}n+4, & \text { for } i=1 \\
2+2 i, & \text { for } 2 \leq i \leq n-1 \\
3 n+2, & \text { for } i=n\end{cases}
\end{aligned}
$$

It is easy to check that there are no two edges of the same weight and there are no two vertices of the same weight. So $\phi$ is a totally irregular total $k$-labeling. We conclude that $t s\left(D_{n}\right)=2 n+1$. Which complete the proof.

\section{The crossed prism graph $C_{n}$}

In [4] A. Ahmad et al. defined the cross prism graph $C_{n}$ and found the vertex irregular total labeling of the cross prism graphs and is denoted by $C_{n}$. The set of vertices and edges of $C_{n}$ is given as followed.

$$
\begin{gathered}
V\left(C_{n}\right)=V\left\{a_{i} ; b_{i}: 1 \leq i \leq n\right\} \\
E\left(C_{n}\right)=\left\{a_{i} a_{i+1} ; b_{i} b_{i+1} ; a_{i} b_{i+1} ; a_{i} b_{i-1}: 1 \leq i \leq n\right\} \cup\left\{a_{1} b_{n} ; a_{n} b_{1}\right\} .
\end{gathered}
$$

In the next theorem we determined the total irregularity strength of crossed prism graphs $C_{n}$. 


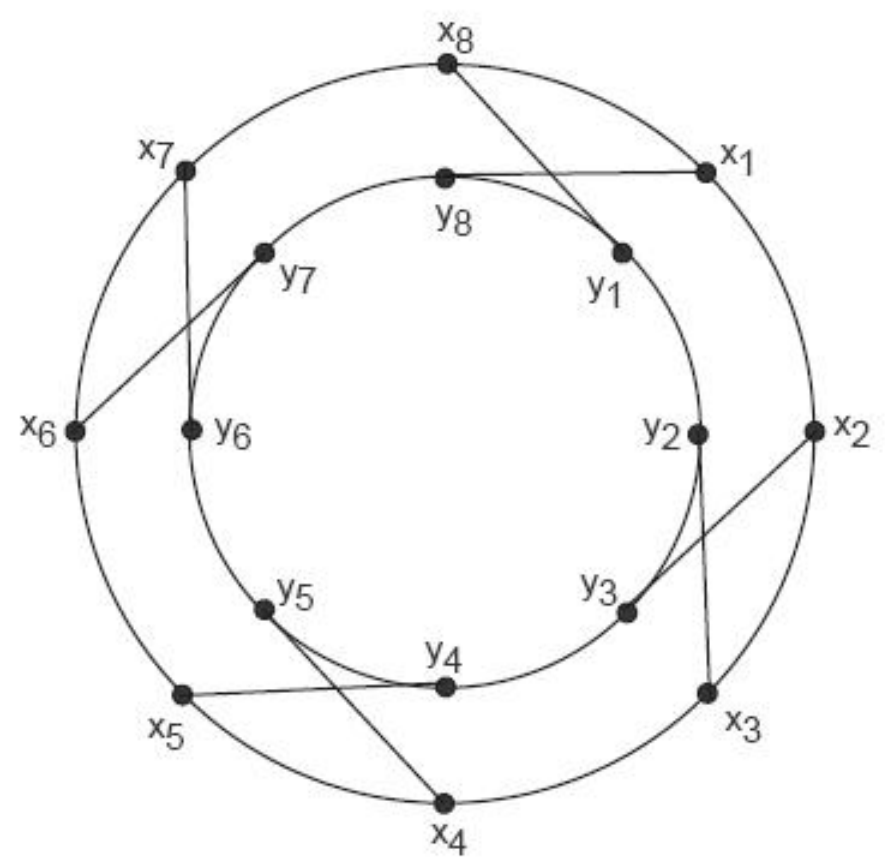

Figure 2: The cross prism graph $C_{8}$

Theorem 4. Let $C_{n}, n \geq 4$ and $n$ is even be a crossed prism graph, Then $\operatorname{ts}\left(C_{n}\right)=n+1$

Proof: Since $\left|E\left(C_{n}\right)\right|=3 n$, so from Theorem 1, tes $\left(C_{n}\right) \geq n+1$. Also $C_{n}$ has $2 n$ vertices of degree 3 , so from Theorem 2, we get $\operatorname{tvs}\left(C_{n}\right) \geq$ $\left\lceil\frac{2 n+3}{4}\right\rceil$. From equation (1.1), we get $\operatorname{ts}\left(C_{n}\right) \geq n+1$ Now we show that $t s\left(C_{n}\right) \leq n+1$ For this we define a total labeling $\phi$ from $V\left(C_{n}\right) \cup E\left(C_{n}\right)$ into $\{1,2, \ldots, n+1\}$ and compute the vertex weight and edge weight in the following way.

Let $k=n+1$ and $1 \leq i \leq n$.

$\phi\left(b_{i}\right)=1, \phi\left(a_{i}\right)=k, \phi\left(b_{i} b_{i+1}\right)=i, \phi\left(a_{i} a_{i+1}\right)=i, \phi\left(a_{1} b_{n}\right)=2, \phi\left(a_{n} b_{1}\right)=1$, $w t\left(b_{i} b_{i+1}\right)=2+i, w t\left(a_{i} a_{i+1}\right)=2 n+2+i, w t\left(a_{i}\right)=n+2+i, w t\left(a_{1} b_{n}\right)=$ $n+4, w t\left(a_{n} b_{1}\right)=n+3$, 
Case.1. when $i$ is odd

$\phi\left(a_{i} b_{i-1}\right)=n+3-i, \quad 3 \leq i \leq n-1$, $w t\left(a_{i} b_{i-1}\right)=2 n+5-i, \quad 3 \leq i \leq n-1$, $w t\left(b_{i}\right)=2 n+3+i, \quad 1 \leq i \leq n-1$,

Case.2. when $i$ is even

$\phi\left(a_{i} b_{i+1}\right)=n+1-i, \quad 2 \leq i \leq n-2$, $w t\left(b_{i}\right)=2 n+1+i, \quad 2 \leq i \leq n$, $w t\left(a_{i} b_{i+1}\right)=2 n+3-i, \quad 2 \leq i \leq n-2$,

It is easy to check that there are no two edges of the same weight and there are no two vertices of the same weight. So $\phi$ is a totally irregular total $k$-labeling. We conclude that $t s\left(C_{n}\right)=n+1$. Which complete the proof.

\section{The necklace graph $N_{n}$}

In [4] A. Ahmad et al. defined the necklace graph $N_{n}$ and found the vertex irregular total labeling of $N_{n}$. The necklace graph has $2 n+3$ vertices and having the vertex set and the edge set as follows.

$$
\begin{gathered}
V\left(N_{n}\right)=V\left\{a_{i}: 1 \leq i \leq n\right\} \cup\left\{b_{j}: 2 \leq j \leq n-1\right\} \\
E\left(N_{n}\right)=\left\{a_{i} a_{i+1}: 1 \leq i \leq n-1\right\} \cup\left\{b_{j} b_{j+1}: 2 \leq i \leq n-2\right\} \cup\left\{a_{i} b_{j}: 2 \leq i, j \leq n-1:\right\} \\
\cup\left\{a_{1} a_{n}, a_{1} b_{2}, a_{n} b_{n-1}\right\}
\end{gathered}
$$

where the subscript $n+1$ must be replaced by 1 .

In the next theorem we determined the total irregularity strength of necklace graph $N_{n}$. 


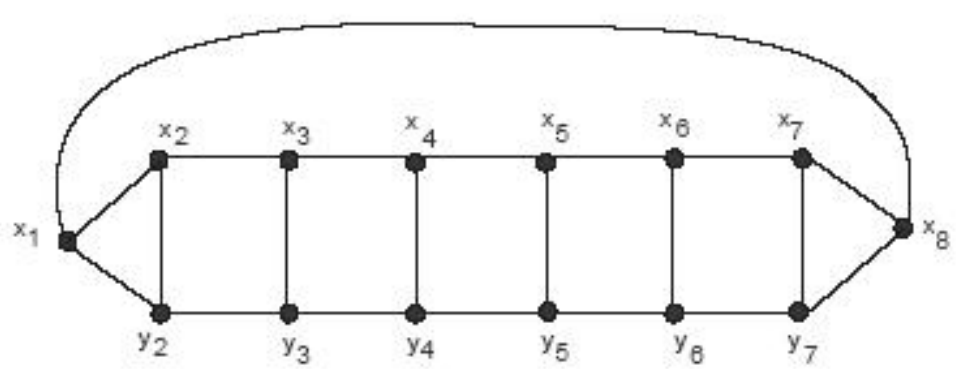

Figure 3: The necklace graph $N_{8}$

Theorem 5. Let $N_{n}, n \geq 4$ be necklace graph, Then $t s\left(N_{n}\right)=n$.

Proof: Since $\left|E\left(N_{n}\right)\right|=3 n-3$, so from Theorem 1 tes $\left(N_{n}\right) \geq n$. Also $N_{n}$ has $2 n-2$ vertices of degree 3 , so from Theorem 2 , we get $\operatorname{tvs}\left(N_{n}\right) \geq\left\lceil\frac{2 n+1}{4}\right\rceil$. From equation (1.1), we get $t s\left(N_{n}\right) \geq n$. Now we show that $t s\left(N_{n}\right) \leq n$. For this we define a total labeling $\phi$ from $V\left(N_{n}\right) \cup E\left(N_{n}\right)$ into $\{1,2, \ldots, n\}$ and compute the vertex weight and edge weights in the following way. $\phi\left(b_{j} b_{j+1}\right)=j, \quad 2 \leq j \leq n-2$ $\phi\left(a_{i} b_{j}\right)=n+2-i, \quad 2 \leq i, j \leq n-1$ $\phi\left(b_{j}\right)=1, \quad 2 \leq j \leq n-1$, $\phi\left(a_{1} a_{n}\right)=2, \phi\left(a_{n} b_{n-1}\right)=1, \phi\left(a_{1} b_{2}\right)=1$,

$$
\begin{gathered}
\phi\left(a_{i} a_{i+1}\right)= \begin{cases}1+i, & \text { for } 1 \leq i \leq n-3 \\
n, & \text { for } i=n-2, n-1\end{cases} \\
\phi\left(a_{i}\right)= \begin{cases}1, & \text { for } i=1 \\
n, & \text { for } 2 \leq i \leq n-1 \\
n-1, & \text { for } i=n\end{cases}
\end{gathered}
$$

$w t\left(b_{j} b_{j+1}\right)=2+j, \quad 2 \leq j \leq n-2$ $w t\left(a_{i} b_{j}\right)=2 n+3-i, \quad 2 \leq i, j \leq n-1$, $w t\left(a_{1} b_{2}\right)=3, w t\left(a_{1} a_{n}\right)=n+2, w t\left(a_{n} b_{n-1}\right)=n+1$,

$$
w t\left(a_{i} a_{i+1}\right)= \begin{cases}n+3, & \text { for } i=1 \\ 2 n+1+i, & \text { for } 2 \leq i \leq n-3 \\ 3 n, & \text { for } i=n-2 \\ 3 n-1, & \text { for } i=n-1\end{cases}
$$




$$
\begin{aligned}
& w t\left(a_{i}\right)= \begin{cases}6, & \text { for } i=1 \\
2 n+3+i, & \text { for } 2 \leq i \leq n-3 \\
3 n+2, & \text { for } i=n-2 \\
3 n+3, & \text { for } i=n-1 \\
2 n+2, & \text { for } i=n\end{cases} \\
& w t\left(b_{j}\right)= \begin{cases}n+2+j, & \text { for } 2 \leq j \leq n-2 \\
n+3, & \text { for } j=n-1\end{cases}
\end{aligned}
$$

It is easy to check that there are no two edges of the same weight and there are no two vertices of the same weight. So $\phi$ is a totally irregular total $k$-labeling. We conclude that $\operatorname{ts}\left(N_{n}\right)=n$. Which complete the proof.

\section{The goldberg snark graph $G_{n}$}

The goldberg snark graph $G_{n}$ is a 3 regular graph with $12 n$ vertices denoted by $G_{n}$ is a graph with the vertex set and the edge set as follows.

$$
V\left(G_{n}\right)=V\left\{a_{i} ; b_{i} ; c_{i} ; d_{i} ; e_{i} ; f_{i} ; g_{i} ; h_{i} ; 1 \leq i \leq n\right\}
$$

$E\left(G_{n}\right)=\left\{a_{i} a_{i+1} ; e_{i+1} f_{i} ; g_{i} h_{i+1} ; a_{i} b_{i} ; b_{i} c_{i} ; b_{i} d_{i} ; c_{i} e_{i} ; d_{i} f_{i} ; e_{i} f_{i} ; c_{i} g_{i} ; d_{i} h_{i} ; g_{i} h_{i} 1 \leq\right.$ $i \leq n\}$

where the subscript $n+1$ must be replaced by 1 .

In the next theorem we determined the total irregularity strength of goldberg snark graph $G_{n}$. 


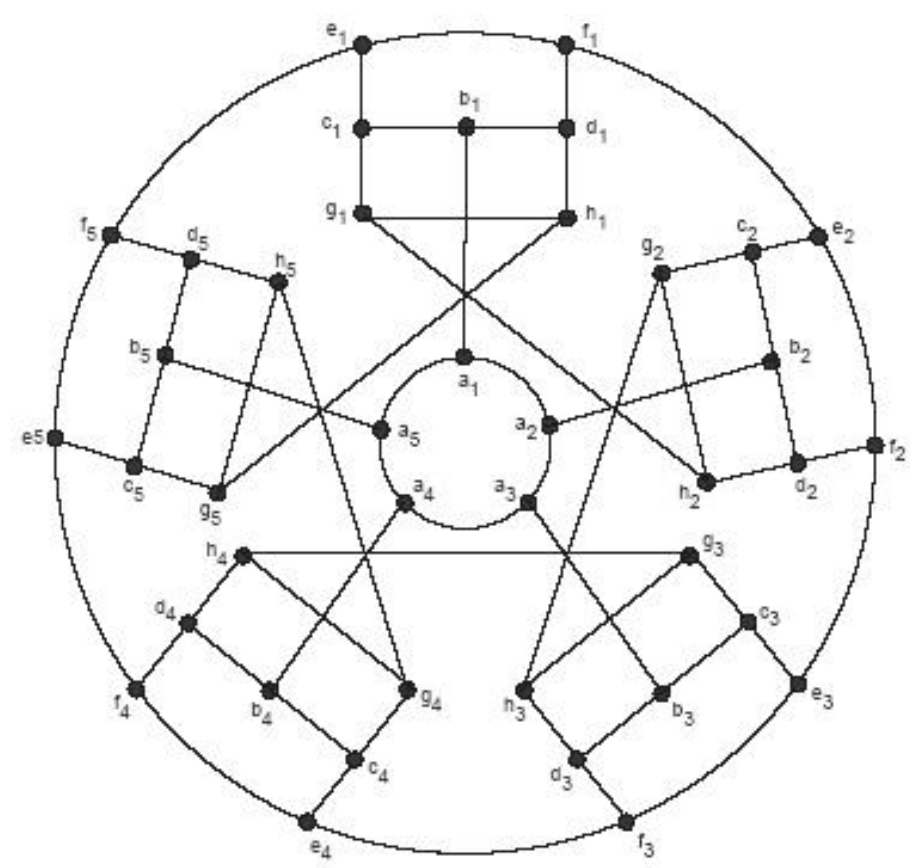

Figure 4: The goldberg Snark graph $G_{5}$

Theorem 6. Let $G_{n}, n \geq 3$ be goldberg snark graph, Then $t s\left(G_{n}\right)=$ $\left\lceil\frac{12 n+2}{3}\right\rceil=4 n+1$.

Proof: Since $\left|E\left(G_{n}\right)\right|=12 n$, so from Theorem 1, tes $\left(G_{n}\right) \geq 4 n+1$. Also $G_{n}$ has $8 n$ vertices of degree 3, so from Theorem 2 , we get $\operatorname{tvs}\left(G_{n}\right) \geq$ $\left\lceil\frac{8 n+3}{4}\right]$. From equation (1.1), we get $t s\left(G_{n}\right) \geq 4 n+1$. Now we show that $t s\left(G_{n}\right) \leq 4 n+1$. For this we define a total labeling $\phi$ from $V\left(G_{n}\right) \cup E\left(G_{n}\right)$ into $\{1,2, \ldots, 4 n+1\}$ and compute the vertex weight and edge weights in the following way.

Let $k=4 n+1$ and $1 \leq i \leq n$,

$\phi\left(a_{i}\right)=k, \phi\left(b_{i}\right)=k, \phi\left(c_{i}\right)=2 n-1+2 i, \phi\left(d_{i}\right)=2 n+2 i, \phi\left(e_{i}\right)=1$, $\phi\left(f_{i}\right)=1, \phi\left(g_{i}\right)=2 n+1, \phi\left(h_{i}\right)=2 n+1, \phi\left(a_{i} a_{i+1}\right)=k-i, \phi\left(a_{i} b_{i}\right)=2 n+i$, $\phi\left(b_{i} c_{i}\right)=2 n+1, \phi\left(b_{i} d_{i}\right)=2 n+1, \phi\left(c_{i} e_{i}\right)=1, \phi\left(d_{i} f_{i}\right)=1, \phi\left(c_{i} g_{i}\right)=1$, $\phi\left(d_{i} h_{i}\right)=1$, 


$$
\begin{aligned}
& \phi\left(e_{i} f_{i}\right)= \begin{cases}2, & \text { for } i=1 \\
2 i-1, & \text { for } 2 \leq i \leq n\end{cases} \\
& \phi\left(g_{i} h_{i}\right)= \begin{cases}2 n+2, & \text { for } i=1 \\
2 n-1+2 i, & \text { for } 2 \leq i \leq n\end{cases} \\
& \phi\left(e_{i+1} f_{i}\right)= \begin{cases}1, & \text { for } i=1 \\
2 i, & \text { for } 2 \leq i \leq n\end{cases} \\
& \phi\left(g_{i} h_{i+1}\right)= \begin{cases}2 n+1, & \text { for } i=1 \\
2 n+2 i, & \text { for } 2 \leq i \leq n\end{cases}
\end{aligned}
$$

$$
\begin{gathered}
w t\left(e_{i} f_{i}\right)= \begin{cases}4, & \text { for } i=1 \\
2 i+1, & \text { for } 2 \leq i \leq n\end{cases} \\
w t\left(g_{i} h_{i}\right)= \begin{cases}6 n+4, & \text { for } i=1 \\
6 n+1+2 i, & \text { for } 2 \leq i \leq n\end{cases} \\
w t\left(e_{i+1} f_{i}\right)= \begin{cases}3, & \text { for } i=1 \\
2+2 i, & \text { for } 2 \leq i \leq n\end{cases} \\
w t\left(g_{i} h_{i+1}\right)= \begin{cases}6 n+3, & \text { for } i=1 \\
6 n+2+2 i, & \text { for } 2 \leq i \leq n\end{cases} \\
w t\left(a_{i}\right)= \begin{cases}13 n+3, & \text { for } i=1 \\
14 n+4-i, & \text { for } 2 \leq i \leq n\end{cases} \\
w t\left(e_{i}\right)= \begin{cases}2 n+4, & \text { for } i=1 \\
6, & \text { for } i=2 \\
4 i-1, & \text { for } 3 \leq i \leq n\end{cases} \\
w t\left(h_{i}\right)= \begin{cases}8 n+4, & \text { for } i=1 \\
6 n+6, & \text { for } i=2 \\
6 n-1+4 i, & \text { for } 3 \leq i \leq n\end{cases}
\end{gathered}
$$

It is easy to check that there are no two edges of the same weight and there are no two vertices of the same weight. So $\phi$ is a totally irregular total $k$-labeling. We conclude that $t s\left(G_{n}\right)=\left\lceil\frac{12 n+2}{3}\right\rceil$. Which complete the proof. 


\section{References}

[1] A. Ahmad, M. Bača, and M. K. Siddiqui, "On edge irregular total labeling of categorical product of two cycles", Theory of computing systems, vol. 54, no. 1, pp. 1-12, 2014, doi:10.1007/ s00224-013-9470-3

[2] A. Ahmad, M. Ibrahim, and M. K. Siddiqui, "On the total irregularity strength of Generalized Petersen Graph", Mathematical reports, vol. 18, no. 2, pp. 197-204. [On line]. Available: https:/ / bit.ly/ 36JNCNq

[3] A. Ahmad,M. Bača, Y. Bashir and M. K. Siddiqui, "Total edge irregularity strength of strong product of two paths", Ars combinatoria, vol. 106, pp. 449-459, 2012. [On line]. Available: https:/ / bit.ly/ 3hSccSO

[4] A. Ahmad, S. A. Bokhari, M. Imran, and A. Q. Baig, "Vertex irregular total labeling of cubic graphs", Utilitas mathematica, vol. 91, pp. 287-299, 2013. [On line]. Available: https:/ / bit.ly/ 3irw0v9

[5] O. Al-Mushayt, A. Ahmad, and M. K. Siddiqui, "On the total edge irregularity strength of hexagonal grid graphs", Australasian journal of combinatorics, vol. 53, pp. 263-271, 2012. [On line]. Available: https:/ / bit.ly/ 2VZpCnr

[6] A. Ahmad, O. Al Mushayt, and M. K. Siddiqui, "Total edge irregularity strength of strong product of cycles and paths", Scientific Bulletin "Politehnica" University of Bucharest. Series A, Applied mathematics and physics (Online), vol. 76, no. 4, pp. 147-156, 2014. [On line]. Available: https:/ / bit.ly/ 2UUAEK4

[7] A. Ahmad, M. K. Siddiqui, and D. Afzal, "On the total edge irregularity strength of zigzag graphs", Australasian journal of combinatorics, vol. 54, pp. 141-149, 2012. [On line]. Available: https:/ / bit.ly/ 3wLjjAw

[8] M. Anholcer and C. Palmer, "Irregular labelings of circulant graphs", Discrete mathematics, vol. 312, no. 23, pp. 3461-3466, 2012, doi: 10.1016/j.disc.2012.06.017

[9] M. Bača, S. Jendro'l, M. Miller, and J. Ryan, "On irregular total labellings", Discrete mathematics, vol. 307, no. 11-12, pp. 1378-1388, 2007, doi: $10.1016 /$ j.disc.2005.11.075

[10] M. Bača, and M. K. Siddiqui, "Total edge irregularity strength of generalized prism", Applied mathematics and computation, vol. 235, pp. 168-173, 2014, doi: 10.1016/j.amc.2014.03.001 
[11] G. Chartrand, M. S. Jacobson, J. Lehel, O. R. Oellermann, S. Ruiz, and F. Saba, "Irregular networks", Congressus numerantium, vol. 64, 1988, 187-192. [On line]. Available: https:/ / bit.ly/ 2UrP9oS

[12] C. Tong, L. Xiaohui, Y. Yuansheng, and W. Liping, "Irregular total labellings of $\mathrm{Cm} \square \mathrm{Cn}$ ”, Utilitas mathematica, vol. 81, pp. 3-13, 2010.

[13] C. Tong, L. Xiaohui, Y. Yuansheng, and W. Liping, "Irregular total labellings of some families of graphs", Indian journal of pure and applied mathematics, vol. 40, no. 3, pp. 155-181, 2009. [On line]. Available: https:/ / bit.ly/ 3xVxMez

[14] J. H. Dimitz, D. K. Garnick and A. Gyárfás, “On the irregularity strength of the $\mathrm{m} \times \mathrm{n}$ grid", Journal of graph theory, vol. 16, no. 4, pp. 355-374, 1992, doi: 10.1002/ jgt.3190160409

[15] J. Ivančo and S. Jendro'l, "Total edge irregularity strength of trees", Discussiones mathematicae graph theory, vol. 26, no. 3, pp. 449-456, 2006, doi: 10.7151/ dmgt.1337

[16] S. Jendro'l, J. Miškuf, and R. Soták, "Total edge irregularity strength of complete and complete bipartite graphs", Electronic notes in discrete mathematics, vol. 28, pp. 281-285, 2007, doi: 10.1016/j.endm. 2007.01.041

[17] S. Jendro'l, J. Miškuf, and R. Soták, "Total edge irregularity strength of complete graphs and complete bipartite graphs", Discrete mathematics, vol. 310, no. 3, pp. 400-407, 2010, doi: 10.1016/j.disc.2009.03.006

[18] C. C. Marzuki, A. N. M. Salman, and M. Miller, "On the total irregularity strength on cycles and paths", Far East journal of mathematical sciences, vol. 82, no. 1, pp. 1-21, 2013.

[19] J. Miškuf and S. Jendro'l, “On total edge irregularity strength of the grids", Tatra mountains mathematical publications, vol. 36, pp. 147-151, 2007.

[20] Nurdin, E. T. Baskoro, A. N. M. Salman and N. N. Gaos, "On the total vertex irregularity strength of trees", Discrete mathematics, vol. 310, no. 21, pp. 3043-3048, 2010, doi: 10.1016/j.disc.2010.06.041

[21] R. Ramdani and A. N. M. Salman, "On the total irregularity strength of some cartesian product graphs", AKCE International journal of graphs and combinatorics, vol. 10, no. 2, pp. 199-209, 2013, doi: 10.1080/ 09728600.2013.12088736 